\title{
Robotic Animals Might Aid in the Social Development of Children with Autism
}

\author{
Cady M. Stanton ${ }^{1}$, Peter H. Kahn, Jr. ${ }^{1}$, Rachel L. Severson ${ }^{1}$, Jolina H. Ruckert ${ }^{1}$, \& Brian T. Gill ${ }^{2}$ \\ ${ }^{1}$ Department of Psychology \\ University of Washington \\ Seattle, Washington, USA \\ ${ }^{2}$ Department of Mathematics \\ Seattle Pacific University \\ Seattle, Washington, USA \\ cadyms [pkahn; raches; jhr333] @ \\ u.washington.edu \\ bgill@spu.edu
}

\begin{abstract}
This study investigated whether a robotic dog might aid in the social development of children with autism. Eleven children diagnosed with autism (ages 5-8) interacted with the robotic dog AIBO and, during a different period within the same experimental session, a simple mechanical toy dog (Kasha), which had no ability to detect or respond to its physical or social environment. Results showed that, in comparison to Kasha, the children spoke more words to AIBO, and more often engaged in three types of behavior with AIBO typical of children without autism: verbal engagement, reciprocal interaction, and authentic interaction. In addition, we found suggestive evidence (with $p$ values ranging from .07 to .09 ) that the children interacted more with AIBO, and, while in the AIBO session, engaged in fewer autistic behaviors. Discussion focuses on why robotic animals might benefit children with autism.
\end{abstract}

\section{Categories and Subject Descriptors}

I.2.9 [Artificial Intelligence]: Robotics - commercial robots and applications. J.4 [Social and Behavioral Sciences]: psychology. K.4.2 [Computers and Society]: Social Issues assistive technologies for persons with disabilities.

\section{General Terms}

Experimentation, Human Factors.

\section{Keywords}

Autism, Robots, AIBO, Social Development, Reciprocity, Animals.

\section{INTRODUCTION}

Researchers within HRI have begun to explore the potential of robots to aid children with autism. This paper contributes to this nascent body of research. We report on a study of children with autism interacting with Sony's robotic dog AIBO.

\footnotetext{
Permission to make digital or hard copies of all or part of this work for personal or classroom use is granted without fee provided that copies are not made or distributed for profit or commercial advantage and that copies bear this notice and the full citation on the first page. To copy otherwise, or republish, to post on servers or to redistribute to lists, requires prior specific permission and/or a fee.

HRI'08, March 12-15, 2008, Amsterdam, Netherlands.

Copyright 2008 ACM 978-1-60558-017-3/08/03...\$5.00.
}

Autism is a pervasive developmental disorder characterized by three main symptoms $[5,13,28]$. The first - and perhaps most significant - is impaired social interaction. Compared to their typically developing peers, children with autism focus their attention more on objects, such as toys, and interact much less with other people. This lack of interaction leaves children with autism with poor social skills, which in turn sets into motion a cycle where their awkward attempts at social interaction meet with negative feedback from other children and adults alike, which leads to further social withdrawal. The second symptom is impaired communication. Approximately $30 \%$ of individuals with autism lack spoken language. Children who can speak are likely to demonstrate atypical mannerisms, such as immediate or delayed echolalia, which is the tendency to mimic others' speech rather than forming their own sentences. Other atypical verbal patterns include unusual intonation, syntax, and word choice. The third symptom is the presence of repetitive behaviors and restricted interests. Repetitive behaviors may take the form of repeated motor movements, such as hand-flapping, toe-walking, spinning in circles or even self-injurious behaviors, as well as persistently using objects in a non-functional, repetitive manner (e.g., spinning the wheels on a toy car for an hour).

There have been some pioneering studies in HRI that suggest that robots might be able to ameliorate one or more of these symptoms $[3,4,6,14-17,22,24-27,30-32]$. A large body of this research was initiated and continues to be carried forward by Dautenhahn, Robins, and their colleagues. For example, they conducted a number of studies of children with autism interacting with a mobile non-humanoid robot. They found that children were not afraid of the robot, and were more attracted to the mobile robot than a non-robotic toy. In addition, some of the children with autism used the robot as a medium for social interaction with another child, and there were instances where children used the robot as the means to make contact with an adult (for a summary, see [4]). In other studies, Dautenhahn, Robins, and their colleagues used a humanoid robotic doll "Robota." In one longitudinal study, for example, they investigated the interactions of four children with autism interacting with Robota over a period of several months. Two of the four children showed an increase across sessions in their overall level of interaction with the robot, as measured by the quantity of time spent looking at the robot, being in close proximity to the robot, touching the robot, and imitating the robot. Qualitative results were also suggestive. For example, one boy (with no verbal language) responded to Robota's movements with a dance of his own. Six months after the study 
was completed, the boy again encountered Robota, and again responded with a dance. The researchers speculated that this dance was the child's attempt to communicate with the robot. Along related lines, Kozima and colleagues have provided suggestive evidence with two different types of robots - an upper-torso humanoid robot [14] and a small creature-like robot Keepon [15] - that robots have the potential to engage children socially and provide a pivotal role for enhanced communication with adults.

The current study sought to extend this emerging body of research in three ways. First, while researchers have been clearly aware that the robot form matters greatly in human-robot interaction [18, 19], except for one study [6], which focused more on technical issues of the robot itself, we know of no research that has investigated children with autism interacting with a robotic non-human animal. Yet in the autism literature, animals have been shown to be effective in increasing social interaction and communication in this population. For example, in one study [29], 22 children with autism, over the course of 15 weeks, participated in both a weekly traditional occupational therapy session, and one that incorporated animals. In the sessions incorporating animals, children interacted more socially and used more language. In another study [20], children with autism in the presence of a dog were more likely to demonstrate a happy, playful mood, and interacted socially with the dog, both looking at it and talking to it. Moreover, in the presence of a dog the children's communications with the therapist were more likely to be on-topic and interactive.

Thus for our robot in the current study we sought a dog form. Previous research by Kahn and his colleagues has investigated children's and adults' interactions with and reasoning about the robotic dog AIBO. In one study, for example, they investigated preschool children's reasoning and behavior in relation to AIBO and a stuffed dog as a comparison artifact [10]. In a second study, they investigated older children's and adolescents' reasoning and behavior in relation to AIBO and a real dog as a comparison [21]. In a third study, they investigated the social discourse of adults in online AIBO discussion forums [7]. Taken together, these studies provide evidence that people (children and adults) establish social and in some limited ways even moral relationships with AIBO. Accordingly, AIBO seemed a good choice as the robot to use in our current investigation.

The second way we sought to extend the emerging body of HRI research of children with autism was by comparing children's social interactions with AIBO to a mechanical but non-robotic dog (we named Kasha). The main difference between the two artifacts was that unlike AIBO, Kasha had no ability to detect or respond to its physical or social environment. Thus this current study directly investigated whether the seemingly autonomous, self-directing, and self-organizing features of AIBO could account for improved social interaction in children with autism.

Third, we sought to deepen and coalesce a set of measures for assessing child-robot social interaction of children with autism. In our study, children interacted with both AIBO and Kasha during different times within the same experimental session. During each session, an experimenter was present and sought to engage the child in some social conversation and with each of the artifacts. We then measured four aspects of social interaction that are central to the autism literature and HRI literature discussed above. The first was the amount of time children spent interacting with each artifact (AIBO and Kasha). The second was the amount of speech each child produced in talking to each artifact. The third was the number of behavioral social interactions typical of children without autism that each child engaged in with each artifact. And the fourth was the number of behavioral social interactions typical of children with autism that each child engaged in with each artifact.

Although this study was exploratory, we expected to find evidence that AIBO, as compared to Kasha, engaged children more as a social other, and facilitated greater social interaction with other people.

\section{METHODS}

\subsection{Participants}

Eleven children between the ages of 5 and 8 participated in this study. All participants had a formal diagnosis of autism, as well as some verbal ability. In addition, participants had no significant vision, hearing, or motor impairments; no history of head injury; and no history of neurological disease. The study was open to both males and females, but only one girl enrolled in the study. This gender imbalance likely occurred, in part, because autism is three to four times more common in boys than girls [5]. Participants were recruited from the communities in and around two cities: Yakima, Washington, and Seattle, Washington, USA.

\subsection{Materials}

$A I B O$. In the experimental condition, participants interacted with the robotic dog AIBO (Figure 1, top). AIBO was designed to be an "autonomous robot" $\operatorname{dog}$ [12]. It has a dog-like metallic form, moveable body parts, and sensors that can detect distance, acceleration, vibration, sound, and pressure. As one of its compelling activities, AIBO can locate a pink ball through its image sensor, and walk toward the pink ball, kick it, and head butt it. In somewhat unpredictable patterns, not unlike a live dog, AIBO will shake itself, sit down, lie down, stand up, walk, and rest. AIBO also initiates interactions with humans, such as offering its paw; and it may respond with "pleasure" (green lights) or "displeasure" (red lights) after certain forms of interaction (such as a person shaking its paw or not).

Kasha. In the control condition, participants interacted with a simple toy dog we named Kasha (Figure 1, bottom). It was similar to AIBO in both size and shape. Like AIBO, Kasha could walk, wag its tail, and make noise. As noted above, however, Kasha had no ability to detect or respond to its physical or social environment.

Props and Toys. Additional materials included a bright pink ball and a dog biscuit that were used as props in interacting with the two artifacts. There were also a variety of neutral toys in the room (e.g., a puzzle, plastic blocks, and a squishy ball) to make the area more child-friendly.

\subsection{Design and Procedure}

Each child participated in an individual interactive session with both artifacts in a large room. The presentation order of the two artifacts was counterbalanced. The protocol was designed to be completed within 30 minutes, so as not to overtax the attention 

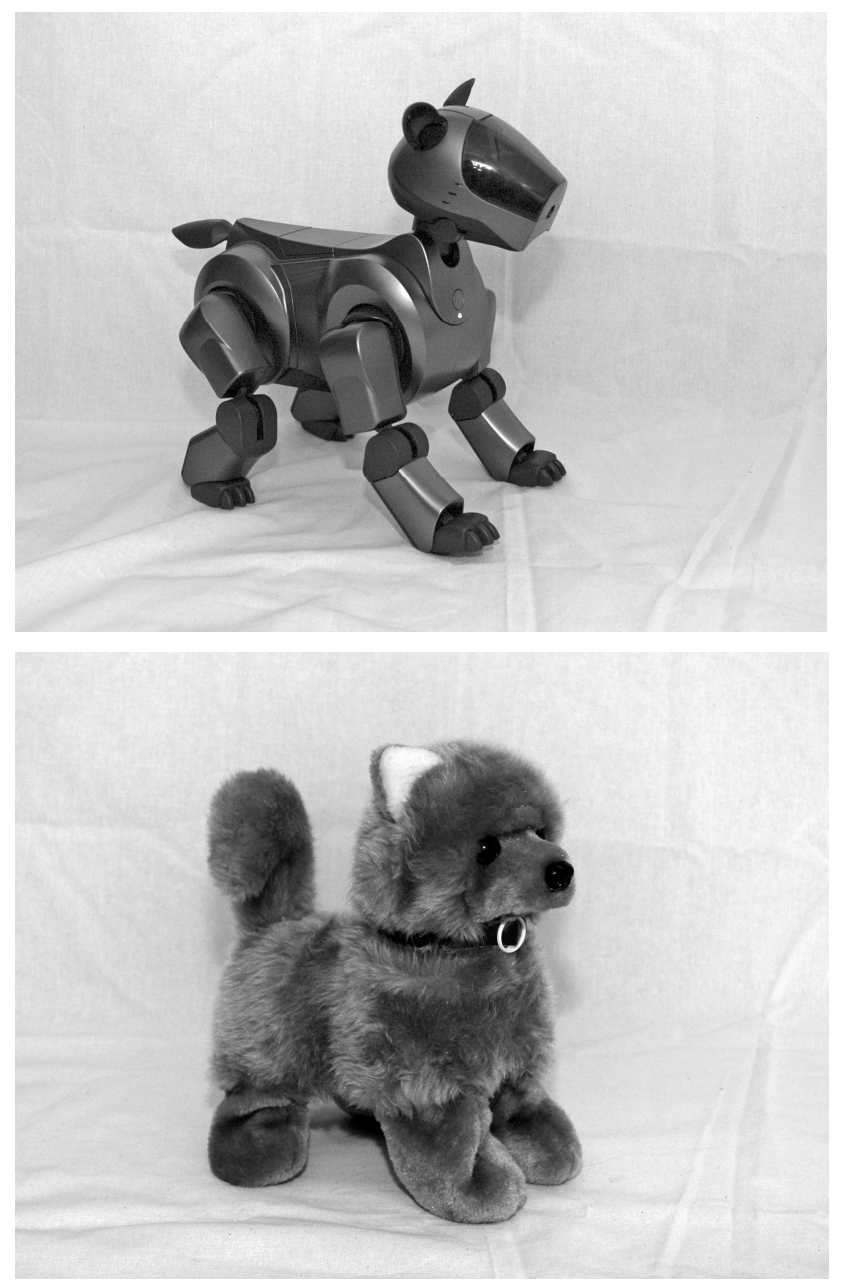

Figure 1. The two artifacts: AIBO (top) and Kasha (bottom).

span of the children. Sessions were videotaped, and the camera person was present in the same room with the child.

Because children with autism often feel anxious in unfamiliar environments and with unfamiliar people, we paid special attention to making the child feel comfortable. For example, each session began with a brief introductory period designed to allow the child to become familiar with and at ease in the room, with the neutral toys on the floor so the child could play with them if desired. Too, the child's parent was present in the room at all times. We asked the parent not to initiate interactions with his or her child, but said it was fine to be responsive if the child initiated such interactions. Once the parent confirmed that the child appeared comfortable in the room, the experimenter introduced the first artifact. At this junction, the experiment formally started.

The experimenter brought out of a cupboard either AIBO or Kasha (depending on the counter-balanced condition), and turned on the artifact within the child's view. The experimenter told the child it was okay to pet the artifact, and asked him if he would like to do so. The experimenter then let the child engage in self-directed exploratory play for a few minutes. Next the experimenter asked a series of pre-established questions, while the child continued to play, and invited the child to engage in certain pre-established behavioral interactions with the artifact, such as holding it and rolling the ball to it. The purpose of the questions and invited behavioral interactions was to provide a consistent structure across sessions, while encouraging social engagement with both the artifact and the experimenter.

\subsection{Coding Categories and Procedures}

Drawing from previous work on typically-developing preschool children's interactions with AIBO [10], a behavioral coding manual was developed from a portion of the data and then applied to the entire data set. The behavioral categories (and subcategories) are listed below. Key coding procedures are also described.

Through our procedures, we sought to deal with a difficult problem in coding behavioral interactions in HRI: knowing when one behavior ends and another begins. For example, as described by Kahn et al. [10], imagine a child petting AIBO by running his hand back and forth along AIBO's body. Should each coupling of a back and forth movement be counted as "one pet"? Or should each unidirectional movement be counted as a pet? Now imagine that the child stops petting for an instant (say, half a second), and then continues petting in the same direction he was moving. Should the movement following the slight pause be counted as the continuation of the initial petting behavior? If so, what if the child stops for one second? Five seconds? Where does a pause indicate a break in one unit of behavior and the start of a new unit of identical behavior? This example illustrates just one of dozens of such difficulties that arose. Thus to establish a reliable means of coding a distinct behavioral unit, we coded a behavior only once within a specified unit of time: either 5 seconds or 30 seconds, depending on the specific behavior. We determined these units by reviewing video footage with the intent to find the best compromise between demarcating behavior cohesively (e.g., not chopping up a clearly single behavior into different timed sections) and increasing the frequency count of the behaviors (and thus increasing the statistical power in our analyses).

Interaction with Artifact. This category captured the time the child was engaged with the artifact, including such activities as the following: touching, talking to, throwing or kicking or offering the ball to, and gesturing to. Eye gaze was not enough to initiate an interaction; however, eye gaze during an on-going interaction was sufficient to maintain that interaction. During a period of interaction, non-interaction could occur for up to five seconds, and still be part of the initial interaction period. If there was a break in the interaction for $5+$ seconds, non-interaction started at the time of the break.

Spoken Communication to Artifact. This category captured the number of meaningful words said by the child to the artifact. Hyphenated words (e.g., uh-huh, choo-choo, chow-chow) counted as one word. If the child made a number of utterances in a row, with only a subset recognizable, then only the number of recognizable words were coded.

Behavioral Interactions with Artifact Typical of Children without Autism. This category comprised five sub-categories derived from Kahn et al. [10] and Kahn et al. [11]: (1) Verbal engagement, including salutations, valedictions, and general conversation. (2) Affection, including petting, scratching, 
tender touching, kissing, and hugging. (3) Animating Artifact, including moving the artifact's body or part of its body (e.g., helping the artifact walk or eat its biscuit), and speaking words or sounds for the artifact (e.g., "woof-woof"). (4) Reciprocal Interaction, including motioning with arms, hands, or fingers to give direction to the artifact, verbal cues (e.g., directives or questions), and offerings (e.g., showing the artifact the dog biscuit or throwing the artifact the ball) - all with some expectation of a response from the artifact. And (5) Authentic Interaction, either dyadic with the artifact or triadic between the child, artifact, and experimenter (e.g., the speed, tone, and volume of the child's voice is exceptionally well modulated for the circumstances, or the child's body is in a state of repose oriented toward the artifact as a social partner). Each behavior was coded only once within a 30 -second period. Thus, for example, if a child petted AIBO more or less continuously for 100 seconds, that behavior would be coded as 4 occurrences of Affection.

Behavioral Interactions with Artifact Typical of Children with Autism. The Gilliam Autism rating scale [8] lists 42 behaviors that are indicative of autism. We reviewed this inventory a priori to coding the data, and pulled out all behaviors (16 in total) that we thought could be found in our data set and could be reliably coded. These behaviors were the following: (1) rocks back and forth, (2) flicks fingers or hands, (3) high-pitched noise, (4) unintelligible sounds, (5) repeats words, (6) lines up objects, (7) prances/walks on tiptoe, (8) whirls/turns in circles, (9) inappropriate pronouns, (10) uses third person for self, (11) withdraws/standoffish, (12) unreasonable fear, (13) licks objects, (14) smells objects, (15) lunging/darting, and (16) selfinjurious. To help the reader understand what we excluded, here are some examples (with our reasons in parentheses): "Avoids asking for things he or she wants" (would require a long-term relationship with the child to determine); "resists physical contact from others" (not appropriate for our data, as we never sought to establish physical contact with the child); "looks through people" (not possible to code reliably with our video data); and "responds inappropriately to simple commands" (we did not consistently offer any commands to the child; also it was a novel situation, so it is not clear what "appropriate" behavior would be). Each behavior was coded only once within a 30 second period.

\subsection{Reliability}

A second individual was trained in the use of the coding manual and recoded 5 of the 11 videos. Intercoder reliability was assessed using Spearman's rank correlation coefficient $\rho$. For percentage of interaction with the artifacts, $\rho=.969$; for number of words per minute to the artifacts, $\rho=.966$; for behavioral interactions typical of children without autism, the average $\rho=$ .732; and for behavioral interaction typical of children with autism, $\rho=.919$.

\section{RESULTS}

To test for differences in children's responses between AIBO and Kasha, results were compared using the Wilcoxon Signed Ranks Test, a non-parametric, within-subject test.

For all 11 children, the session with AIBO $(M=15.4$ minutes, $\mathrm{SD}=3.56)$ was longer than the session with Kasha $(M=10.1$ minutes, $\mathrm{SD}=3.55), Z=-2.93, p=.003$. This finding may be of interest in itself (e.g., it may suggest that children found
AIBO more engaging than Kasha), but it is difficult to interpret with confidence as it could be due to other factors (e.g., experimenter bias). Accordingly, for all relevant tests below we accounted for this effect by focusing on the number of occurrences per minute (of the behavior of interest) rather than the total number of occurrences. In this way, our results can be considered conservative, with stronger effects almost always emerging had we not performed this adjustment.

\subsection{Percentage of Interaction}

We coded for how much time the child spent not interacting (see Figure 2, top) and interacting with each artifact during the session. The results were suggestive. Children spent an average of $72 \%$ of the AIBO session actually interacting with AIBO, and only $52 \%$ of the Kasha session actually interacting with Kasha. The Wilcoxon signed-rank test approached statistical significance: $Z=-1.689, p=.091$.

\subsection{Spoken Communication to Artifact}

We examined the total number of words the children spoke to each of the artifacts. Results showed that the children spoke more words per minute to AIBO $(M=2.73$ words, $\mathrm{SD}=3.05)$ than to Kasha $(M=1.07$ words, $\mathrm{SD}=1.62), Z=-2.073, p=$ .038 .
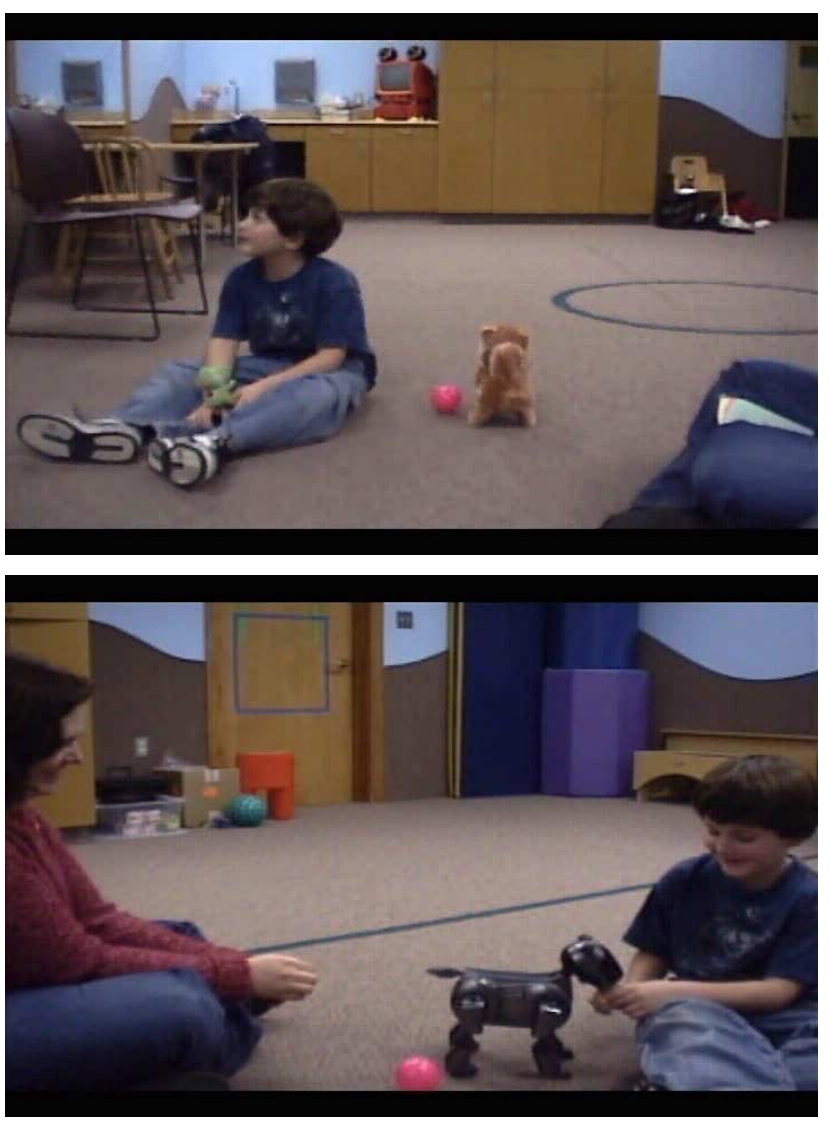

Figure 2. The same participant in "non-interaction" with Kasha (top) and in "authentic interaction" with AIBO (bottom). 
Table 1. Occurrences of Behaviors Typical of Children without Autism by Artifact

\begin{tabular}{|c|c|c|c|c|c|c|c|c|}
\hline \multirow{3}{*}{ Behavioral Interaction Category } & \multirow{2}{*}{\multicolumn{2}{|c|}{$\begin{array}{c}\text { Total Occurrences } \\
\text { (across all } \\
\text { participants) }\end{array}$}} & \multicolumn{4}{|c|}{$\begin{array}{l}\text { Occurrences per Minute } \\
\text { for Each Participant }\end{array}$} & \multicolumn{2}{|c|}{$\begin{array}{c}\text { Wilcoxon } \\
\text { Signed-Rank Test }\end{array}$} \\
\hline & & & \multicolumn{2}{|c|}{ Mean } & \multicolumn{2}{|c|}{ Median } & \multirow{2}{*}{$\begin{array}{c}\text { Test } \\
\text { Stat. }(Z)\end{array}$} & \multirow{2}{*}{$P$-value } \\
\hline & AIBO & Kasha & AIBO & Kasha & AIBO & Kasha & & \\
\hline 1 Verbal Engagement & 27 & 2 & .180 & .034 & .123 & 0 & -2.380 & .017 \\
\hline 2 Affection & 93 & 61 & .598 & .694 & .542 & .260 & -.445 & .657 \\
\hline 3 Animating Artifact & 6 & 42 & .037 & .272 & 0 & 0 & -1.214 & .225 \\
\hline 4 Reciprocal Interaction & 169 & 53 & .970 & .458 & .757 & .489 & -2.134 & .033 \\
\hline 5 Authentic Interaction & 17 & 2 & .105 & .011 & .091 & 0 & -2.666 & .008 \\
\hline
\end{tabular}

\subsection{Behavioral Interactions Typical of Children without Autism}

We coded for five categories of behavioral interactions typical of children without autism: verbal engagement, affection, animating artifact, reciprocal interaction, and authentic interaction. As shown in Table 1, children engaged in more verbal engagement, reciprocal interaction, and authentic interaction with AIBO compared to Kasha. The category authentic interaction (see Figure 2, bottom) was broken down into two subcategories: dyadic (between child and artifact) and triadic (between child, artifact, and experimenter). Results showed both more authentic dyadic interaction $(p=.018)$ and more authentic triadic interaction $(p=.028)$ in the AIBO condition compared to the Kasha condition. There was no difference in the amount of affection or animation that the children accorded AIBO or Kasha. (Note that it might appear from the total occurrences that children animated AIBO less than Kasha, 6 compared to 42 total occurrences, respectively. But this difference was driven by large amounts of animation with Kasha by just three participants, and was not statistically significant.)

\subsection{Behavioral Interactions Typical of Children with Autism}

We coded for 16 behaviors typical of children with autism: (1) rocks back and forth, (2) flicks fingers or hands, (3) high-pitched noise, (4) unintelligible sounds, (5) repeats words, (6) lines up objects, (7) prances/walks on tiptoe; (8) whirls/turns in circles, (9) inappropriate pronouns, (10) uses third person for self, (11) withdraws/standoffish, (12) unreasonable fear, (13) licks objects, (14) smells objects, (15) lunging/darting, and (16) selfinjurious. Results showed no statistically significant differences between AIBO and Kasha in the number of occurrences per minute of any of the individual behaviors. However, when we combined all the behaviors together, results were highly suggestive. Specifically, the mean number of autistic behaviors per minute with AIBO was .75, and the mean number of autistic behaviors per minute with Kasha was 1.1. The Wilcoxon signed-ranked test came close to statistical significance: $Z=$ $1.84, p=.066$.

\section{DISCUSSION}

The results from this study suggest that AIBO, as representative of a robotic dog, might aid in the social development of children with autism. Specifically, in comparison to a mechanical toy dog (which had no ability to detect or respond to its physical or social environment), we found that the children spoke more words to AIBO, and more often engaged in three types of behavior with AIBO typical of children without autism: verbal engagement, reciprocal interaction, and authentic interaction. In addition, we found suggestive evidence that the children interacted more with AIBO, and, while in the AIBO session, engaged in fewer autistic behaviors.

Recruiting children for this study was much harder than in other studies we have conducted with typically-developing children. The upshot was that we had a small sample size (11 participants), and little power in our statistical tests. Nonetheless, we attained statistical significance on the above key measures; and in our view our suggestive findings warrant consideration.

We utilized a new behavioral category in this study: "authentic interaction." We had first conceptualized this category by drawing on the last of nine benchmarks that Kahn et al. [11] have proposed for human-robot interaction: the authenticity of relation. Kahn et al. defined this benchmark using Buber's [1] account of an I-You relationship, wherein an individual relates to another with his or her whole being, freely, fully in the present, unburdened by conceptual knowledge. In turn, given our population of children with autism, we recast this category in somewhat less ethereal terms, and sought to capture what many of us probably take for granted in our social relationships. Namely, in relation to a social partner, we make nuanced adjustments in our facial expressions, body positions, and speech to respond to the other appropriately in context. That was partly how we defined authentic interaction in this study. Quantitatively, that was what we found more of with children interacting with AIBO compared to Kasha.

To convey this new category illustratively, consider the following representative event from our data. (Figure 2, bottom, is a still photo of this event while it was in progress). The child sits on the floor with AIBO and the experimenter. The child is 
trying to convince AIBO to eat the dog biscuit. The child's hand movements are gentle, his body position relaxed. He softly says to AIBO: "open wide, here comes the choo-choo." The experimenter then asks the child if his mother did that with him when he was little, and he giggles and says no, and then adds "I saw it on Viva Piñata." This interaction feels "authentic" insofar as it feels whole, engaged, nuanced, even intimate. The child is integrating gentle behavior with AIBO and appropriate imaginary speech ("open wide, here comes the choo-choo") with an on-topic conversation with the experimenter, drawing on his past experience (of watching Viva Piñata). Contrast that interaction with the same child's interactions with Kasha, in which he looks disengaged (Figure 2, top), and roams around the room and responds distractedly to the experimenter's questions, with answers such as "because she is," "yes," "yeah," and "uhhuh."

Our results support the dual ways in which Kozima, Nakagawa, \& Yasuda [15] and others have suggested that robots might aid in the social development of children with autism. One way is through engaging with the robot as a social other. This proposition was supported by our findings based on children's speech production, verbal engagement, reciprocal interaction, and authentic interaction. Granted, as Robins, Dautenhahn, and Dubowski [23] discuss, it remains an open question whether children's social engagement with a robot transfers to their social engagement with other people. Yet, at least for some forms of functioning, it seems to us that it would. For example, if (as in the current study) children with autism produce more coherent speech with a robot as compared to other play artifacts, such production would seemingly provide the children with increased capability that they could later utilize in human-human interaction. A second way is that robots might provide children with autism with a pivotal medium for enhanced communication with adults. We found support for this proposition insofar as children more often engaged in authentic interaction with the experimenter in the AIBO condition as compared to the Kasha condition.

Our results were based on a robot with an animal (dog) form. Given the success in the autism literature of animal-assisted therapy $[20,29]$ it may be the case that the animal form is a particularly good one for use with this population. Future studies could examine this proposition by drawing on our measures, or other measures, while experimentally employing an animal robot, a humanoid robot, and a mobile (not biologicallike) robot, all three with similar capabilities. It is possible that the humanoid robot would fare the worst because children with autism tend to find human beings overly complex, and thus the humanoid form might trigger in the children with autism similar patterns of interacting. That said, it is also possible that the humanoid robot form offers the greatest potential in helping to move children with autism more fully into social life with other people.

What we saw with some of the children is that, yes, they enjoyed Kasha's repetitive behaviors, and they often engaged Kasha in their own repetitive behaviors (such a repeatedly lining Kasha up on a line on the floor). But we suspect these children recognized something of what we, as the experimenters, recognized: that Kasha's behavior had a dreary sameness to it, compared to AIBO's behavior. Kasha made the exact same sound and moved in the exact same way every single minute it was turned on. In contrast, AIBO's repetitive behavior varied. For example, while AIBO often searched for its pink ball, one never knew when it would do so, and when it did so the pattern varied: the ball was in a new location, AIBO approached it in different ways, oriented itself to the ball in different ways, and was more or less successful with its kick or head butt depending on the many nuanced aspects of its programming. One key factor, then, is this: AIBO is patterned simply but not simply repetitive. In this sense, AIBO is similar to much of nature. People can be captivated, for example, by looking at ocean waves breaking onto the shore, or experiencing a waterfall, because the water's pattern is always changing, simple but compelling [9]. A second key factor is that AIBO, more so than Kasha, exhibits autonomous and goal-directed behavior - social behavior. Both factors together may explain why the children spoke more words to AIBO and engaged with AIBO with more verbal interaction, reciprocal interaction, and authentic interaction.

One limitation of this study is that AIBO differed from Kasha in both form and function, and experimentally we were unable to disambiguate to two. Future studies would profit by moving in this direction. For example, one approach would be to compare an interactive AIBO with a non-interactive AIBO. That said, AIBO's programming does not allow for adding or subtracting behavioral units. Thus this approach would require, in effect, the entire reprogramming of an AIBO: a substantial technical undertaking.

Finally, we would like to discuss a difficult problem that has begun to emerge in HRI of how to conceive of investigations of children with autism. Two approaches have been offered. ${ }^{1}$ One approach builds on the following sort of reasoning: It is first pointed out that autism is a term that currently covers a wide range of disparate (and at times perhaps unrelated) behaviors, each of which can vary widely in terms of its manifest severity. It is then pointed out that the scientific community poorly understands the origins of these behaviors, both in terms of their genetic basis and how the expressions of genetic tendencies are affected by environment and culture. Given this state of affairs, it is then reasoned that generalizable findings of children with autism interacting with robots should not be expected. Rather, investigations with this population of children should be understood more as case studies. It takes something of the form: "Here, let me show you what worked with at least a few of the children in my study. Then you try it, and maybe you'll find that it will work with some other children with autism. Over time, we'll hope to build up a repertoire of techniques, but we shouldn't be expecting any technique to work for this population as a whole."

The other approach responds to such reasoning skeptically. A rebuttal can take the following form: "Imagine we have a clinical theory that proposes that young boys, around age 5, want to sleep with their mother, based on sexual instincts, but that that desire is frustrated by the demands of society, and in particular the father of the family, and thus the young boy feels anger toward the father, hatred actually, and feels the desire to

\footnotetext{
${ }^{1}$ These ideas were developed through an email exchange with Marek Michalowski, 2007, while he was working in the lab of Hideki Kozima.
} 
kill the father, and yet loves the father, and then comes to believe and fear that his father will castrate him, and on and on until we've explicated Freud's full account of the Oedipal Complex. Now let's say that we discover through case studies that on occasion people with neurotic symptoms get somewhat better when we engage them in therapy based on our theory of the Oedipal Complex. Not everyone gets better, of course. But then we say that neurosis is a very broad term covering many types of people and situations and histories, and we shouldn't expect generalizable findings." Have we provided compelling evidence for the theory? Many would say no [2]. Thus this rebuttal pushes on the limitations of the case study methodology, arguing instead for the scientific method and generalizable results.

In our view, both approaches have merit, at least while autism remains poorly understood by psychologists and while the field of HRI is still young. That said, the current study was framed more with the second approach in mind. Namely, we sought and found - results that generalized to our research sample of participants. Thus this study contributes to a literature in HRI that shows that at least some important questions pertaining to children with autism are scientifically tractable.

\section{ACKNOWLEDGMENTS}

We thank Advanced Telecommunications Research, Intelligent Robotics and Communications Labs, and specifically Norihiro Hagita, Hiroshi Ishiguro, and Takayuki Kanda, for use of their debugger software system which we used for reviewing video footage and coding data. We thank Earl Wilson for discussions about autism at various phases in this research. Thanks, too, to Daniel Glick, Heidi Shaw and her students at Yakima Valley Community College, and Children's Village and the Autism Resource Team of Yakima, WA. This research was supported, in part, by a University of Washington Mary Gates Research Scholarship to the first author, and as part of a grant (No. IIS0325035) to the second author by the National Science Foundation. Any opinions, findings, and conclusions or recommendations expressed in this material are those of the authors and do not necessarily reflect the views of the National Science Foundation.

\section{REFERENCES}

[1] Buber, M. I and thou. Touchstone, New York, 1996.

[2] Crews, F. C. The memory wars : Freud's legacy in dispute. New York Review of Books, New York, 1995.

[3] Dautenhahn, K., and Werry, I. Towards interactive robots in autism therapy. Pragmatics and Cognition, 12, 1 (2004), $1-35$.

[4] Dautenhahn, K., Werry, I., Salter, T., and Boekhorst, R. t. Towards adaptive autonomous robots in autism therapy: Varieties of interactions. In Proceedings of the IEEE International Symposium on Computational Intelligence in Robotics and Automation (Kobe, Japan, July 16-20, 2003). Piscataway, NJ: IEEE, 2003, 577-582.

[5] Dawson, G., and Toth, K. Autism spectrum disorders. In D. Cicchetti and D. J. Cohen (Eds.), Developmental psychopathology: Risk, disorder and adaptation (Vol. 3). John Wiley \& Sons, Inc., Hoboken, NJ, 2006, 317-357.
[6] François, D., Polani, D., and Dautenhahn, K. On-line behaviour classification and adaptation to human-robot interaction styles. In Proceedings of the ACM/IEEE international conference on Human-robot interaction (Arlington, Virginia, 2007). New York, NY: ACM Press, 2007, 295-302.

[7] Friedman, B., Kahn, P. H., Jr., and Hagman, J. Hardware companions?: What online AIBO discussion forums reveal about the human-robotic relationship. In Proceedings of the SIGCHI Conference on Human Factors in Computing Systems (Ft. Lauderdale, Florida, USA, April 5-10, 2003). New York, NY: ACM Press, 2003, 273-280.

[8] Gilliam, J. E. The Gilliam Autism Rating Scale. Pro-Ed, Austin, TX, 1995.

[9] Kahn, P. H., Jr. The human relationship with nature: development and culture. MIT Press, Cambridge, Mass., 1999.

[10] Kahn, P. H., Jr., Friedman, B., Pérez-Granados, D. R., and Freier, N. G. Robotic pets in the lives of preschool children. Interaction Studies: Social Behavior and Communication in Biological and Artificial Systems, 7, 3 (2006), 405-436.

[11] Kahn, P. H., Jr., Ishiguro, H., Friedman, B., Kanda, T., Freier, N. G., Severson, R. L., and Miller, J. What is a human? Toward psychological benchmarks in the field of human-robot interaction. Interaction Studies: Social Behavior and Communication in Biological and Artificial Systems, 8, 3 (in press).

[12] Kaplan, F., Oudeyer, P.-Y., Kubinyi, E., and Miklosi, A. Robotic clicker training. Robotics and Autonomous Systems, 38, 3-4 (2002), 197-206.

[13] Koegel, R. L., Koegel, L. K., and McNerney, E. K. Pivotal areas in intervention for autism. Journal of Clinical Child Psychology, 30, 1 (2001), 19-32.

[14] Kozima, H., Nakagawa, C., Kawai, N., Kosugi, D., and Yano, Y. A humanoid in company with children. In Proceedings of the 4th IEEE/RAS International Conference on Humanoid Robots (Los Angeles, CA, November 10-12, 2004). Piscataway, NJ: IEEE, 2004, 470-477.

[15] Kozima, H., Nakagawa, C., and Yasuda, Y. Designing and observing human-robot interactions for the study of social development and its disorders. In Proceedings of the 2005 IEEE International Symposium on Computational Intelligence in Robotics and Automation (Espoo, Finland, June 27-30, 2005). Piscataway, NJ: IEEE, 2005, 41-46.

[16] Kozima, H., and Yano, H. Designing a robot for contingency-detection game. In Proceedings of the Workshop on Robotic and Virtual Interactive Systems in Autism Therapy (Hertfordshire, England, September 27-28, 2001). Hertfordshire, England: University of Hertfordshire, 2001, 35-38.

[17] Liu, C., Conn, K., Sarkar, N., and Stone, W. Affect recognition in robot assisted rehabilitation of children with autism spectrum disorder. In Proceedings of the IEEE International Conference on Robotics and Automation (Roma, Italy, April 10-14, 2007). Piscataway, NJ: IEEE, 2007, 1755-1760. 
[18] MacDorman, K. F. Androids as an experimental apparatus: Why is there an uncanny valley and can we exploit it? In Proceedings of the CogSci 2005 Workshop: Toward Social Mechanisms of Android Science (Stresa, Italy, July 25-26, 2005), 2005, 106-118.

[19] MacDorman, K. F., and Ishiguro, H. The uncanny advantage of using androids in cognitive and social science research. Interaction Studies: Social Behavior and Communication in Biological and Artificial Systems, 7, 3 (2006), 297-337.

[20] Martin, F., and Farnum, J. Animal-assisted therapy for children with pervasive developmental disorders. Western Journal of Nursing Research, 24, 6 (October 1, 2002, 2002), 657-670.

[21] Melson, G. F., Kahn, P. H., Jr., Beck, A. M., Friedman, B., Roberts, T., Garrett, E., and Gill, B. T. Robots as dogs?: children's interactions with the robotic dog AIBO and a live australian shepherd. Journal of Applied Developmental Psychology(in press).

[22] Robins, B., and Dautenhahn, K. The role of the experimenter in HRI research - A case study evaluation of children with autism interacting with a robotic toy. In Proceedings of the 15th IEEE International Symposium on Robot and Human Interactive Communication (Hatfield, England, September 6-8, 2006). Piscataway, NJ: IEEE, 2006, 646-651.

[23] Robins, B., Dautenhahn, K., and Dubowski, J. Robots as isolators or mediators for children with autism? A cautionary tale. In Proceedings of the AISB '05 Symposium on Robot Companions: Hard Problems and Open Challenges in Robot-Human Interaction (Hertfordshire, England, April 12-15, 2005). Hertfordshire, England: University of Hertfordshire, 2005, 82-88.

[24] Robins, B., Dautenhahn, K., te Boekhorst, R., and Billard, A. Effects of repeated exposure to a humanoid robot on children with autism. In S. Keates, J. Clarkson, P. Langdon and P. Robinson (Eds.), Designing a more inclusive world. Springer-Verlag, London, 2004, 225-236.

[25] Robins, B., Dautenhahn, K., te Boekhorst, R., and Billard, A. Robotic assistants in therapy and education of children with autism: Can a small humanoid robot help encourage social interaction skills? Universal Access in the Information Society, 4, 2 (2005), 105-120.

[26] Robins, B., Dautenhahn, K., te Boerkhorst, R., and Billard, A. Robots as assistive technology - does appearance matter? In Proceedings of the 2004 IEEE International Workshop on Robot and Human Interactive Communication (Kurashiki, Okayama, Japan, September 20-22, 2004). Piscataway, NJ: IEEE, 2004, 277-282.

[27] Robins, B., Dickerson, P., Stribling, P., and Dautenhahn, K. Robot-mediated joint attention in children with autism. Interaction Studies: Social Behavior and Communication in Biological and Artificial Systems, 5, 2 (2004), 161-198.

[28] Rogers, S. J. Evidence-based interventions for language development in young children with autism. In T. Charman and W. Stone (Eds.), Social and communication development in autism spectrum disorders: Early identification, diagnosis, and intervention. Guilford Press, New York, 2006, 143-179.

[29] Sams, M. J., Fortney, E. V., and Willenbring, S. Occupational therapy incorporating animals for children with autism: A pilot investigation. American Journal of Occupational Therapy, 60, 3 (2006), 268-274.

[30] Scassellati, B. How social robots will help us to diagnose, treat, and understand autism. In Proceedings of the 12th International Symposium of Robotics Research (San Francisco, CA, October 12-15, 2005), 2005.

[31] Scassellati, B. Quantitative metrics of social response for autism diagnosis. In Proceedings of the 14th IEEE International Workshop on Robot and Human Interactive Communication (Nashville, TN, August 13-15, 2005). Piscataway, NJ: IEEE, 2005.

[32] Shibata, T., Wada, K., Saito, T., and Tanie, K. Human interactive robot for psychological enrichment and therapy. In Proceedings of the AISB '05 Symposium on Robot Companions: Hard Problems and Open Challenges in Robot-Human Interaction (Hertfordshire, England, April 12-15, 2005). Hertfordshire, England: University of Hertfordshire, 2005, 98-109. 